\title{
Factores asociados a las estancias anormalmente prolongadas en las hospitalizaciones por insuficiencia cardiaca en España
}

\author{
Associated factors with unusually long stays in heart \\ failure hospitalizations in Spain
}

\author{
J.M. García Torrecillas ${ }^{1}$, E. Moreno² ${ }^{2}$ I Sánchez-Montesinos ${ }^{3}$, M.C. Lea ${ }^{4}$
}

\section{RESUMEN}

Fundamento. La insuficiencia cardiaca es un proceso de alta prevalencia que origina repetidos ingresos hospitalarios con sobrecarga asistencial e incremento del gasto sanitario. Los objetivos de este trabajo son describir y caracterizar los casos con estancias prolongadas por este síndrome, detectando posibles factores asociados a la misma.

Método. Estudio de cohorte histórica de todos los episodios de personas mayores de 45 años, ingresados por insuficiencia cardiaca en el Sistema Sanitario Público Español en el período 1997-2007. Fuente: 808.229 episodios clasificados como Grupos Relacionados de Diagnóstico 127 y 544, según el Conjunto Mínimo Básico de Datos del Instituto de Información Sanitaria. Se evaluaron variables sociodemográficas (edad, género, comunidad autónoma), clínicas (comorbilidades, complicaciones, tipo de ingreso y alta) y de gestión (estancia, tipo de hospital, reingresos). Se definió estancia anormalmente prolongada aquella que superó el percentil 90 (14 y 16 días, respectivamente), construyéndose un modelo de regresión logística para valorar sus posibles factores asociados.

Resultados. Presentaron estancias anormalmente prolongadas el $11,4 \%$, mostrando inferior edad media y mayor número de diagnósticos y procedimientos, reingresos y mortalidad que el grupo sin estancias prolongadas. Padecer anemia, insuficiencia renal, TEP o ictus así como el reingreso y el ingreso programado se asociaron a mayor probabilidad de estancia anormalmente prolongada.

Conclusión. Es posible definir un perfil de comorbilidad y sociodemográfico que valore la probabilidad de tener un ingreso prolongado, si bien dadas las características de las bases de datos administrativas la capacidad discriminativa del modelo es discreta.

Palabras clave. Insuficiencia cardíaca, Estancias. Grupos relacionados de diagnóstico. Comorbilidades. Gestión. Urgencias.

\begin{abstract}
Background. Heart failure is a process of high prevalence that causes repeated hospital admissions with increased health care costs. The aim of this article is to describe and characterize the cases with long stays due to this syndrome, identifying associated factors wherever possible.

Method. An historical cohort of all the episodes of people over 45 years with a diagnosis of heart failure admitted in the Spanish Public Health System in the period 1997-2007. Source: 808,229 episodes classified as Diagnosis Related Groups 127 and 544 according to the Minimum Basic Data provided by the Institute for Health Information. We assessed sociodemographic variables (age, gender, region), clinical variables (comorbidities, complications, type of admission and discharge) and management variables (length of stay, type of hospital readmissions). An abnormally prolonged stay (APS) was defined as one exceeding the 90th percentile (14 and 16 days, respectively); we built a logistic regression model to assess their possible associated factors.

Results. Eleven point four percent (11.4\%) presented abnormally prolonged stays, showing lower mean age and increased number of diagnoses and procedures, readmissions and mortality than the non-abnormally prolonged stay group. Anemia, kidney failure, pulmonary embolism or stroke as well as readmission and scheduled admission were associated with increased likelihood of APS.
\end{abstract}

Conclusion. It is possible to define a comorbidities and sociodemographic profile to assess the likelihood of a prolonged hospital stay, but given the nature of administrative database the model's discriminative ability is quite discreet. Key words. Heart failure. Length of stay. Diagnosis related groups. Comorbidities. Management. Emergencies.

\section{Correspondencia}

Juan Manuel García Torrecillas

Complejo Hospitalario Torrecárdenas

Servicio de Cuidados Críticos y Urgencias

Paraje Torrecárdenas, $\mathbf{s} / \mathbf{n}$

04009 Almería

E-mail. garcia.torrec@yahoo.es

4. Servicio de Medicina Interna. Empresa Pública

Hospital de Poniente (El Ejido).

Recepción: 4 de febrero de 2011

Aceptación provisional: 17 de marzo de 2011

Aceptación definitiva: 13 de mayo de 2011 


\section{INTRODUCCIÓN}

La insuficiencia cardíaca (IC) es un proceso de alta prevalencia en la actualidad y un problema de salud pública que alcanza proporciones de epidemia ${ }^{1,2}$, y que conduce a un deterioro progresivo y grave del paciente, similar al ocasionado por las patologías tumorales ${ }^{3}$. Este síndrome presenta un número absoluto de casos en un continuo creciente, supone la causa más frecuente de hospitalización -especialmente a través de acceso urgente- en los mayores de 65 años y provocó el $5 \%$ del total de ingresos en España en 20074,5. La mejora en el pronóstico de la IC ha hecho que, actualmente, se haya pasado de considerarla un equivalente de las enfermedades malignas en cuanto a pronóstico vital, a situarla en el margen de las patologías crónicas. Por ello se hace necesario planificar respuestas en relación a su gestión y desarrollar estrategias eficaces, al ser un elemento que tensiona constantemente el empleo de recursos hospitalarios y la balanza del gasto sanitario, pues la estancia se comporta como una variable proxy del coste directo de las hospitalizaciones ${ }^{6}$.

El objetivo de este estudio es describir las características de aquellos pacientes que presentan hospitalizaciones con estancias muy superiores a la media, y específicamente detectar factores asociados a una mayor probabilidad de sufrir una hospitalización prolongada en el caso de pacientes dados de alta vivos.

\section{MATERIAL Y MÉTODOS}

Se ha realizado un estudio observacional analítico del tipo cohorte retrospectiva, a partir de todos los episodios de hospitalización de pacientes mayores de 45 años generados en el Sistema Sanitario Público Español (SSPE) en el periodo 1997-2007. La fuente de información de los casos se basó en el Conjunto Mínimo Básico de Datos (CMBD), siendo seleccionados todos los episodios de hospitalización correspondientes a los grupos relacionados de diagnóstico (GRD) 127 (IC y shock, peso relativo 1,485 ) y 544 (IC crónica y arritmias cardía- cas con complicaciones mayores, peso relativo 3,478 ), para pacientes mayores de 45 años y en el periodo temporal 1997 a 2007. Los datos fueron facilitados por el Instituto de Información Sanitaria del Ministerio de Sanidad, Igualdad y Política Social ${ }^{7}$, y los mismos no permiten en ningún caso la identificación de los pacientes en aras de la protección e intimidad de los mismos.

Se analizaron variables sociodemográficas (edad, género, comunidad autónoma), clínicas (número de diagnósticos al alta -NDA-, número de procedimientos -NPAy número de procedimientos depurados -NPA_dep-, que corresponden a aquellos casos que tienen, como mínimo un procedimiento codificado; estas variables se consideraron proxy de comorbilidades y complicaciones. Se analizó además el tipo de ingreso -urgente o programado-, tipo de alta -exitus, traslado a domicilio, otro hospital o centro sociosanitario-, así como variables de gestión (estancia, tipo de hospital y reingreso). La mortalidad estudiada es la producida durante el ingreso hospitalario y el reingreso es considerado cuando ocurre en los 30 días siguientes al ingreso, siempre que el mismo no se produzca en una anualidad diferente.

Se realizó una depuración inicial de los datos, eliminando aquellos casos que se comportaron como marginales, según la fórmula $\mathrm{T} 2=\mathrm{Q} 3+1,5(\mathrm{Q} 3-\mathrm{Q} 1)$, donde $\mathrm{Q}$ eran los cuartiles tercero y primero, y $\mathrm{T} 2$ un valor máximo de la estancia definido para los GRD, por encima de los cuales fueron considerados extremos. Una vez eliminados dichos marginales, se determinó como variable dependiente principal la estancia superior al percentil 90 en cada uno de los GRD, esto correspondió a estancias superiores a 14 días en el caso del GRD 127, y la superior a 16 días en el del GRD 544, como expresión de punto de corte para estancias anormalmente prolongadas (EP).

Se realizó un estudio descriptivo, expresando las variables cuantitativas como medias y desviaciones típicas, y las cualitativas mediante tablas de frecuencias y porcentajes, acompañando estos de sus correspondientes intervalos de confianza al $95 \%$, 
calculados por el método binomial exacto en el caso de las variables dicotómicas. Las comparaciones entre las primeras se realizaron mediante el test de Student y para las segundas se empleó el test de $\chi^{2}$ de Pearson.

El análisis bivariado se realizó sobre tres grandes categorías: pacientes con estancias prolongadas dados de alta vivos (EP_vivos), pacientes con estancias no prolongadas dados de alta vivos (NoEP_vivos) y exitus con cualquier duración de estancia a fin de minimizar el sesgo que suponen aquellos pacientes con alta gravedad al ingreso y que fallecen a los pocos días de iniciarse la hospitalización. Se realizaron análisis estratificados para estudiar por una parte si existían diferencias dentro de la categoría EP_vivos en función del GRD al que estaban adscritos los casos y por otra para detectar asociaciones entre la posibilidad de ser EP_vivos y el resto de covariables de interés. También fueron analizadas de manera específica las distintas comorbilidades en función de las tres categorías al alta mencionadas previamente. Finalmente, se desarrolló un modelo de regresión logística multivariante para detectar factores asociados a la probabilidad de tener un ingreso del tipo EP_vivos, siendo seleccionadas aquellas variables que mostraron resultados significativos en el análisis bivariado previo, y excluidas aquellas que, pese a tener significación estadística debido al alto volumen muestral de casos, no presentaban relevancia clínica lo suficientemente importante para justificar su inclusión. En el desarrollo del modelo multivariante se utilizó el procedimiento enter para forzar la entrada de algunas covariables imprescindibles. Finalmente se determinó la capacidad discriminativa del modelo mediante el área bajo la curva, así como la calibración del mismo mediante el test de Hosmer-Lemeshow. Se estimó la significación para $\mathrm{p}$ $<0,05$ y se utilizaron los paquetes estadísticos SPSS versión $15,0^{8}$ y Epidat 3.1.

\section{RESULTADOS}

Se analizaron 808.229 episodios, correspondientes a pacientes mayores de 45 años de ambos GRD, en el periodo 19972207. Presentaron EP 92.358 (11,4\%) episodios de IC, 9,8\% del GRD 127 y 15,4\% del GRD $544\left(\chi^{2}=5119,37, \mathrm{p}<0,001\right)$. La proporción de episodios de cada uno de los GRD investigados, a lo largo de los 11 años, tendía hacia el aumento progresivo del GRD 544 (más complejo) en detrimento del 127 (menor coste y complejidad): este último supuso el 82,5\% de casos en 1997 y el $64,9 \%$ en 2007 , mientras el 544 constituyó, respectivamente, el 17,5 y el $35,1 \%$, disminuyendo asimismo el porcentaje global (13,7\% en $1997,10,5 \%$ en 2007$)$ aunque aumentando en números absolutos los episodios de EP (7.334 en 1997, 10.092 en 2007).

\section{Descripción general de la muestra y comparación en base a dos categorías de alta: EP vs NoEP}

La edad media de los episodios del grupo EP fue de 76,89 (DE 9,69) años frente a 77,10 (DE 9,80) en el grupo NoEP ( $p<0,001$ ), siendo el $56,4 \%$ mujeres y el $43,6 \%$ varones $\left(\chi^{2}=4,30, p<0,001\right)$; la distribución por sexos fue similar en el grupo NoEP $(56,0 \%$ en mujeres y $44,0 \%$ en varones). El 11,3\% de los ingresos urgentes (IU) tuvo EP frente al $13,6 \%$ de los programados (IP) $\left(\chi^{2}=257,04\right.$, $\mathrm{p}<0,0001)$. Los hospitales con un mayor volumen de camas para hospitalización mostraron una mayor probabilidad de emitir altas con EP, presentando los de nivel IV (>1000 camas) una OR de 1,49 (IC 95\%:1,46$1,52)$ respecto a la OR de 1 establecida como referencia en los hospitales de nivel I ( $<200$ camas).

La proporción de reingresos fue algo más elevada en el grupo EP (11,4 vs $10,6 \%$, $\left.\chi^{2}=58,51, \mathrm{p}<0,001\right)$ e independiente del sexo y del GRD, sin embargo se detectó una menor proporción de exitus en el grupo EP $\left(9,6 \%\right.$ vs $\left.10,4 \%, \chi^{2}=61,93, p<0,001\right)$, y se comprobó que los pacientes con mayores estancias tenían, con más frecuencia, destinos a unidades de crónicos y residencias asistidas (3\% de los EP se trasladaron a otros centros frente a $2,4 \%$ de los NoEP $\chi^{2}=313,33 \mathrm{p}<0,001-$ ). 
En el análisis de los diferentes grupos etarios, se observó un crecimiento discreto y paulatino de EP con una distribución de morfología normal, de moda en los 6674 años y suave descenso hasta alcanzar valores menos acusados a medida que progresaba la edad. Hasta el intervalo de 71-75, las mujeres presentaron una mayor proporción de EP que los varones (tomando como denominador el total de episodios para cada sexo y grupo etario); a partir de los 76 años los varones presentan porcentajes de EP superiores a las mujeres. Los episodios con EP tuvieron una edad mínimamente inferior al resto, y presentaron un mayor NDA y NPA que los NoEP.

\section{Descripción de las comorbilidades en el grupo EP}

Las arritmias (especialmente fibrilación auricular -FA-), hipertensión arterial
(HTA), diabetes mellitus y cardiopatía isquémica (CI) fueron las comorbilidades más frecuentes en el grupo EP. La HTA presentó una mayor prevalencia en el sexo femenino ( $49 \%$ vs $35,7 \%, \mathrm{p}<0,001$ ) y la $\mathrm{CI}$ en los varones $(32,1 \%$ vs $20,9 \%, \mathrm{p}<0,001)$ (Fig. 1). En el período analizado, se observó incremento de EPOC (19,9\% en el primer año considerado frente a un $20,2 \%$ en el último), CI (23,4\%vs $26,2 \%)$, HTA $(32,5 \%$ vs $48,9 \%)$, valvulopatías ( $18,7 \%$ vs $28,6 \%$ ), arritmias ( $41,5 \%$ vs $58,4 \%)$, FA en particular (34,5\% vs $50,5 \%)$, insuficiencia renal -IR( $13,5 \%$ vs $21,8 \%)$, anemia ( $11,3 \%$ vs $23,9 \%)$, diabetes $(26,2 \%$ vs $37,6 \%)$ y dislipemia ( $4,0 \%$ vs $17,5 \%)$, mientras las miocardiopatías $(10,3 \%$ vs $10,8 \%)$, los ictus $(4,3 \%$ vs $6,3 \%)$ y las cardiopatías congénitas $(0,3 \%$ vs $0,4 \%$ ) se incrementaron más levemente (Fig. 2). El 93,6\% de los episodios del GRD 127 ingresaron de forma urgente frente al 93,2\% de los del GRD 544.

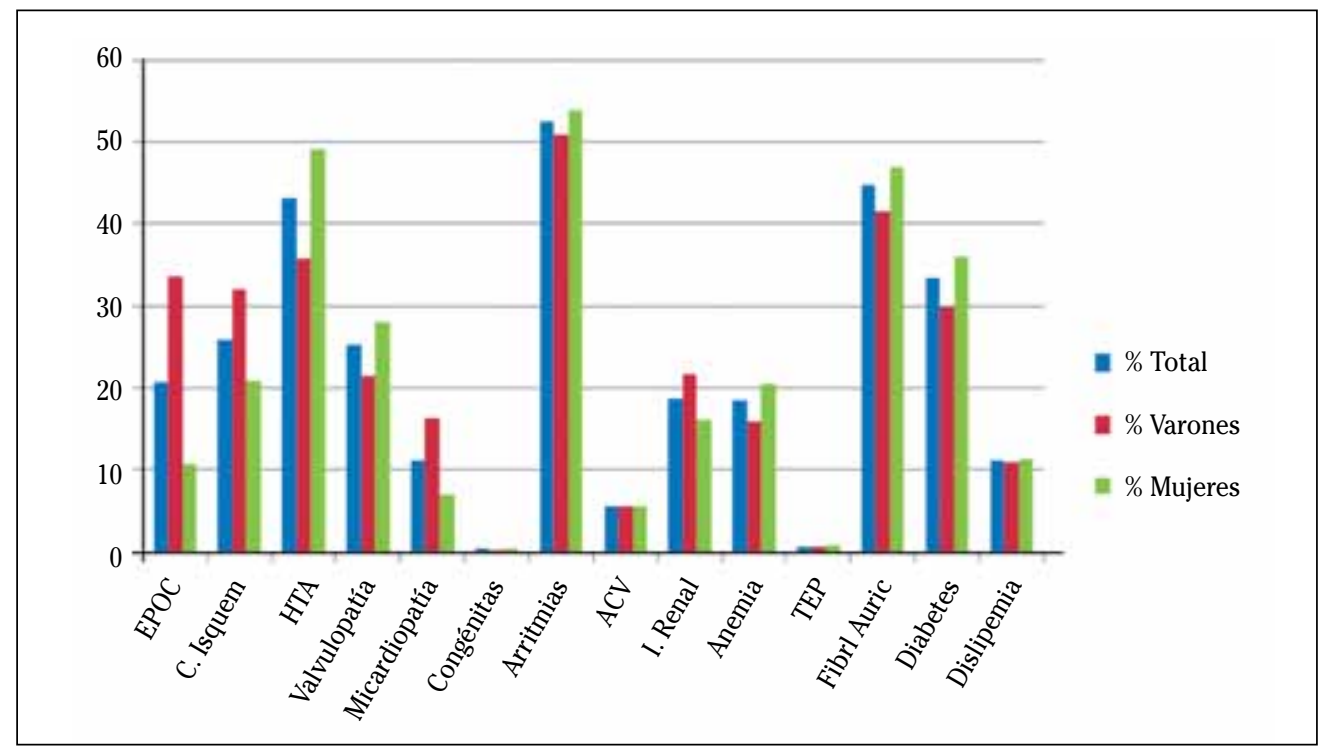

Figura 1. Distribución de comorbilidades por sexos dentro del grupo EP. 

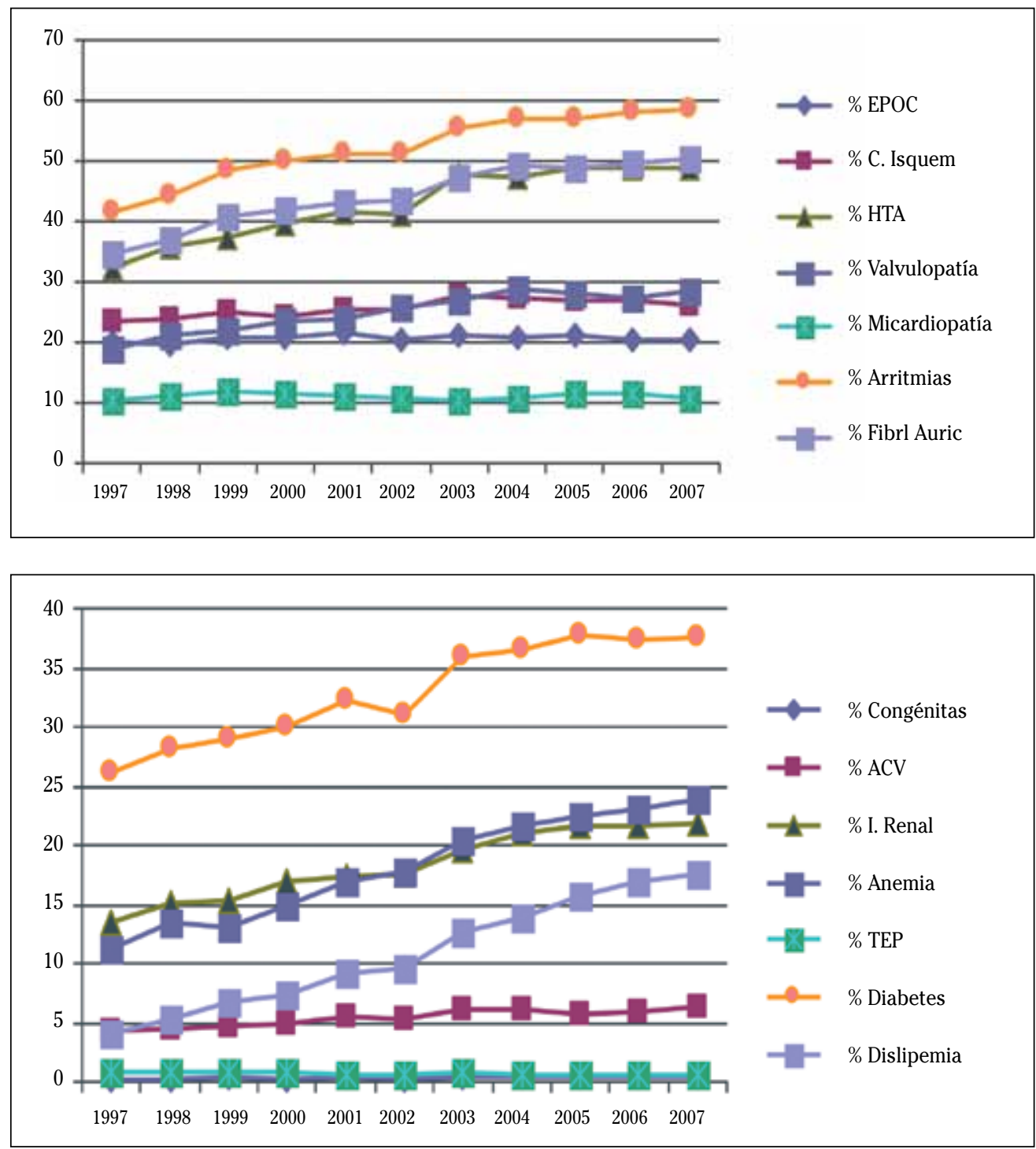

Figura 2. Evolución de las comorbilidades-etiologías (arriba) y precipitantes (abajo) en el período 1997-2007.

\section{Diferencias según GRD dentro del grupo EP (Tabla 1)}

No se evidenciaron diferencias por GRD en cuanto al sexo y proporción de reingresos. Hasta los 75 años existe una mayor proporción de casos asignados al GRD127, no encontramos diferencias en el quinquenio 75-80 y desde aquí hasta los últimos segmentos etarios la proporción de pacientes asignados al GRD más complejo (544) fue significativamente superior.

$\mathrm{Al}$ valorar las diferencias en NDA entre ambos GRD se observó un aumento de las mismas a medida que los diagnósticos y procedimientos aumentaban en número a excepción del último grupo (>6 diagnósticos) donde se acentuó pero se invirtió el 
sentido de las diferencias. El mismo análisis para el NPA mostró un comportamiento errático en relación con la gran variabilidad de la codificación procedimental. Las comorbilidades que mostraron una mayor frecuencia de presentación en el GRD 544 fueron la EPOC, TEP, anemia, IR, arritmias e ictus, siendo más prevalentes en el GRD 127 el resto de las estudiadas. Los resultados se muestran con detalle en la tabla 1 .

Tabla 1. Diferencias por GRD de las principales variables estudiadas en el grupo EP

\begin{tabular}{|c|c|c|c|c|c|c|c|c|c|}
\hline & & \multicolumn{2}{|c|}{ GRD 127} & \multicolumn{2}{|c|}{ GRD 544} & \multicolumn{4}{|c|}{ Diferencias } \\
\hline & & $\mathbf{n}$ & $\%$ & $\mathbf{n}$ & $\%$ & $\%$ & $\begin{array}{c}\mathrm{Li} \\
\text { IC95\% }\end{array}$ & LsIC95\% & p \\
\hline \multirow[t]{2}{*}{ Sexo } & Mujer & 41222 & 56,48 & 16145 & 56,08 & 0,004 & $-0,003$ & 0,011 & 0,25 \\
\hline & Hombre & 31762 & 43,52 & 12643 & 43,92 & $-0,004$ & $-0,011$ & 0,003 & 0,25 \\
\hline \multirow[t]{11}{*}{ Edad, años } & $45-50$ & 954 & 1,31 & 300 & 1,04 & 0,003 & 0,001 & 0,004 & 0,001 \\
\hline & $51-55$ & 1520 & 2,08 & 487 & 1,69 & 0,004 & 0,002 & 0,006 & $<0,0001$ \\
\hline & $56-60$ & 2614 & 3,58 & 795 & 2,76 & 0,008 & 0,006 & 0,011 & $<0,0001$ \\
\hline & $61-65$ & 4497 & 6,16 & 1473 & 5,12 & 0,010 & 0,007 & 0,014 & $<0,0001$ \\
\hline & $66-70$ & 8300 & 11,37 & 2782 & 9,66 & 0,002 & 0,013 & 0,021 & $<0,0001$ \\
\hline & $71-75$ & 12948 & 17,74 & 4543 & 15,78 & 0,020 & 0,015 & 0,025 & $<0,0001$ \\
\hline & $76-80$ & 15670 & 21,47 & 6108 & 21,22 & 0,003 & $-0,003$ & 0,008 & 0,382 \\
\hline & $81-85$ & 14397 & 19,72 & 6122 & 21,27 & $-0,016$ & $-0,021$ & $-0,010$ & $<0,0001$ \\
\hline & $86-90$ & 8717 & 11,94 & 4208 & 14,62 & $-0,003$ & $-0,031$ & $-0,022$ & $<0,0001$ \\
\hline & $91-95$ & 2851 & 3,91 & 1673 & 5,81 & $-0,002$ & $-0,022$ & $-0,016$ & $<0,0001$ \\
\hline & $>95$ & 521 & 0,71 & 297 & 1,03 & $-0,005$ & $-0,005$ & $-0,002$ & $<0,0001$ \\
\hline \multirow[t]{6}{*}{ Ingreso Grupo Hospital } & Urgente & 68286 & 93,76 & 26838 & 93,29 & 0,004 & 0,001 & 0,008 & 0,006 \\
\hline & Programado & 4546 & 6,24 & 1930 & 6,71 & $-0,005$ & $-0,008$ & $-0,001$ & 0,006 \\
\hline & I & 12232 & 16,77 & 4301 & 14,95 & 0,018 & 0,013 & 0,023 & $<0,0001$ \\
\hline & II & 21927 & 30,05 & 9175 & 31,88 & $-0,018$ & $-0,025$ & $-0,012$ & $<0,0001$ \\
\hline & III & 22186 & 30,41 & 8813 & 30,63 & $-0,002$ & $-0,009$ & $-0,004$ & 0,495 \\
\hline & IV & 16622 & 22,78 & 6487 & 22,54 & 0,002 & $-0,003$ & 0,008 & 0,421 \\
\hline \multirow[t]{2}{*}{ Reingreso } & No & 64694 & 88,63 & 25443 & 88,38 & 0,003 & $-0,002$ & 0,0070 & 0,255 \\
\hline & Sí & 8295 & 11,37 & 3345 & 11,62 & 0,003 & $-0,007$ & 0,0020 & 0,255 \\
\hline \multirow[t]{4}{*}{ № Diagnósticos } & $0-1$ & 872 & 1,19 & 0 & 0,00 & 0,012 & 0,011 & 0,013 & $<0,0001$ \\
\hline & $2-3$ & 5407 & 7,41 & 570 & 1,98 & 0,054 & 0,052 & 0,057 & $<0,0001$ \\
\hline & $4-6$ & 29897 & 40,96 & 7602 & 26,41 & 0,146 & 0,139 & 0,152 & $<0,0001$ \\
\hline & $>6$ & 36813 & 50,44 & 20616 & 71,61 & $-0,212$ & $-0,218$ & $-0,205$ & $<0,0001$ \\
\hline
\end{tabular}




\begin{tabular}{|c|c|c|c|c|c|c|c|c|c|}
\hline & & \multicolumn{2}{|c|}{ GRD 127} & \multicolumn{2}{|c|}{ GRD 544} & \multicolumn{4}{|c|}{ Diferencias } \\
\hline & & $\mathbf{n}$ & $\%$ & $\mathbf{n}$ & $\%$ & $\%$ & $\underset{\text { IC95\% }}{\mathbf{L i}}$ & LsIC95\% & $\mathbf{p}$ \\
\hline \multirow[t]{4}{*}{ № Procedimientos } & $0-1$ & 34774 & 47,64 & 10143 & 35,23 & 0,124 & 0,117 & 0,131 & $<0,0001$ \\
\hline & $2-3$ & 19164 & 26,26 & 7361 & 25,57 & 0,007 & 0,001 & 0,013 & 0,025 \\
\hline & $4-5$ & 11266 & 15,44 & 5483 & 19,05 & $-0,036$ & $-0,041$ & $-0,031$ & $<0,0001$ \\
\hline & $>5$ & 7785 & 10,67 & 5801 & 20,15 & $-0,095$ & $-0,1$ & $-0,09$ & $<0,0001$ \\
\hline EPOC & & 12902 & 17,70 & 7295 & 25,30 & $-0,082$ & $-0,071$ & $-0,082$ & $<0,0001$ \\
\hline HTA & & 32051 & 43,90 & 12167 & 42,30 & 0,01 & 0,023 & 0,01 & $<0,0001$ \\
\hline Valvulopatías & & 19905 & 27,27 & 6428 & 22,33 & 0,044 & 0,055 & 0,044 & $<0,0001$ \\
\hline Diabetes & & 25089 & 34,37 & 9008 & 31,29 & 0,024 & 0,037 & 0,024 & $<0,0001$ \\
\hline TEP & & 8 & 0,01 & 533 & 1,85 & $-0,02$ & $-0,017$ & $-0,02$ & $<0,0001$ \\
\hline Anemia & & 13113 & 17,96 & 5354 & 18,60 & $-0,001$ & $-0,001$ & $-0,001$ & 0,019 \\
\hline Insuf. renal & & 9320 & 12,77 & 7942 & 27,59 & $-0,154$ & $-0,142$ & $-0,154$ & $<0,0001$ \\
\hline Arritmias & & 36881 & 50,53 & 16257 & 56,47 & $-0,066$ & $-0,053$ & $-0,066$ & $<0,0001$ \\
\hline Ictus & & 3144 & 4,31 & 2138 & 7,43 & $-0,035$ & $-0,028$ & $-0,035$ & $<0,0001$ \\
\hline Dislipemia & & 8497 & 11,64 & 3086 & 10,72 & 0,005 & 0,014 & 0,005 & $<0,0001$ \\
\hline C. isquémica & & 19958 & 27,34 & 6757 & 23,47 & 0,033 & 0,045 & 0,033 & $<0,0001$ \\
\hline
\end{tabular}

TEP: tromboembolismo pulmonar; Li IC95\%: límite inferior del intervalo de confianza al 95\%; Ls IC95\%: límite superior del intervalo de confianza al 95\% [cálculo mediante el método binomial exacto].

\section{Características de los pacientes EP vivos y factores asociados. Análisis estratificado}

Tal como observamos en la tabla 2 se encuentran diferencias para la probabilidad de ser EP_vivos y el GRD al que estaban adscritos los pacientes, presentando los varones una menor proporción de EP que las mujeres. Considerando la edad en quiquenios la probabilidad de ser EP aumenta hasta los 70 años disminuyendo posteriormente pero ya de modo no significativo a partir de esta edad. Se asoció a ser EP el IP, pertenecer a hospitales de los grupos III y IV y tener más de 2 diagnósticos y/o procedimientos al alta codificados. 
Tabla 2. Características de los pacientes y factores asociados a la probabilidad de tener estancias prolongadas y ser dados de alta vivos. Análisis bivariado

\begin{tabular}{|c|c|c|c|c|c|c|c|c|}
\hline & & \multicolumn{2}{|c|}{ Total (vivos) } & \multicolumn{5}{|c|}{ EP_vivos } \\
\hline & & $\mathbf{n}$ & $\%$ & $\mathbf{n}$ & $\%$ & $\mathrm{OR}$ & IC 95\% & $\mathbf{p}$ \\
\hline \multirow[t]{2}{*}{ GRD } & 127 & 537528 & 74,20 & 68030 & 12,70 & 1 & & \\
\hline & 544 & 187270 & 25,80 & 23955 & 12,80 & 1,01 & 0,99-1,03 & 0,13 \\
\hline \multirow[t]{2}{*}{ Sexo } & Mujer & 405258 & 55,90 & 52007 & 12,80 & 1 & & \\
\hline & Hombre & 319490 & 44,10 & 39973 & 12,50 & 0,97 & $0,96-0,99$ & $<0,0001$ \\
\hline \multirow[t]{11}{*}{ Edad, años } & $45-50$ & 9710 & 1,34 & 1199 & 1,30 & 1 & & \\
\hline & $51-55$ & 15201 & 2,10 & 1921 & 2,09 & 1,03 & $0,95-1,1$ & 0,50 \\
\hline & $56-60$ & 24939 & 3,44 & 3228 & 3,51 & 1,06 & $0,98-1,13$ & 0,14 \\
\hline & $61-65$ & 42548 & 5,87 & 5608 & 6,10 & 1,08 & $1,01-1,15$ & 0,03 \\
\hline & $66-70$ & 77581 & 10,70 & 10314 & 11,21 & 1,09 & $1,02-1,16$ & 0,01 \\
\hline & $71-75$ & 122032 & 16,84 & 16163 & 17,57 & 1,08 & $1,02-1,15$ & 0,01 \\
\hline & $76-80$ & 153149 & 21,13 & 19690 & 21,41 & 1,05 & $0,98-1,12$ & 0,15 \\
\hline & $81-85$ & 145897 & 20,13 & 18245 & 19,83 & 1,01 & $0,95-1,08$ & 0,65 \\
\hline & $86-90$ & 93738 & 12,93 & 11223 & 12,20 & 0,97 & 0,91-1,03 & 0,28 \\
\hline & $91-95$ & 33976 & 4,69 & 3752 & 4,08 & 0,88 & $0,82-0,94$ & 0,00 \\
\hline & $>95$ & 6027 & 0,83 & 642 & 0,70 & 0,85 & $0,76-0,94$ & 0,00 \\
\hline \multirow[t]{2}{*}{ Tipo de ingreso } & Urgente & 684635 & 94,46 & 85930 & 93,42 & 1 & & \\
\hline & Programado & 39432 & 5,44 & 5887 & 6,40 & 1,22 & $1,19-1,26$ & $<0,0001$ \\
\hline \multirow{4}{*}{$\begin{array}{c}\text { Grupo } \\
\text { Hospital }\end{array}$} & I & 138140 & 19,06 & 14779 & 16,07 & 1 & & \\
\hline & II & 247309 & 34,12 & 28041 & 30,48 & 1,07 & $1,05-1,09$ & $<0,0001$ \\
\hline & III & 203854 & 28,13 & 28125 & 30,58 & 1,34 & $1,31-1,37$ & $<0,0001$ \\
\hline & IV & 135364 & 18,68 & 21006 & 22,84 & 1,53 & $1,50-1,57$ & $<0,0001$ \\
\hline \multirow[t]{2}{*}{ Reingreso } & No & 650111 & 89,70 & 81801 & 88,93 & 1 & & \\
\hline & Sí & 74687 & 10,30 & 10184 & 11,07 & 1,09 & $1,07-1,12$ & $<0,0001$ \\
\hline \multirow[t]{4}{*}{ № Diagnósticos } & $0-1$ & 8192 & 1,13 & 686 & 0,75 & 1 & & \\
\hline & $2-3$ & 67993 & 9,38 & 5214 & 5,67 & 0,91 & $0,84-0,99$ & 0,02 \\
\hline & $4-6$ & 306852 & 42,34 & 33993 & 36,95 & 1,37 & $1,26-1,47$ & $<0,0001$ \\
\hline & $>6$ & 341761 & 47,15 & 52092 & 56,63 & 1,97 & $1,82-2,13$ & $<0,0001$ \\
\hline \multirow[t]{4}{*}{ № Procedimientos } & $0-1$ & 415834 & 57,37 & 39867 & 43,34 & 1 & & \\
\hline & $2-3$ & 163487 & 22,56 & 24422 & 26,55 & 1,66 & $1,63-1,68$ & $<0,0001$ \\
\hline & $4-5$ & 87758 & 12,11 & 15363 & 16,70 & 2 & $1,96-2,04$ & $<0,0001$ \\
\hline & $>5$ & 57719 & 7,96 & 12333 & 13,41 & 2,57 & $2,51-2,62$ & $<0,0001$ \\
\hline
\end{tabular}




\begin{tabular}{|c|c|c|c|c|c|c|c|c|}
\hline & & \multicolumn{2}{|c|}{ Total (vivos) } & \multicolumn{5}{|c|}{ EP_vivos } \\
\hline & & $\mathbf{n}$ & $\%$ & $\mathbf{n}$ & $\%$ & OR & IC $95 \%$ & p \\
\hline \multirow[t]{2}{*}{ ЕРОС } & No & 587041 & 80,99 & 73700 & 80,12 & 1 & & \\
\hline & Sí & 137757 & 19,01 & 18285 & 19,88 & 1,07 & $1,05-1,08$ & $<0,0001$ \\
\hline \multirow[t]{2}{*}{ HTA } & No & 369797 & 51,02 & 51185 & 55,64 & 1 & & \\
\hline & Sí & 355001 & 48,98 & 40800 & 44,36 & 0,81 & $0,79-0,82$ & $<0,0001$ \\
\hline \multirow[t]{2}{*}{ Valvulopatías } & No & 549238 & 75,78 & 67810 & 73,72 & 1 & & \\
\hline & Sí & 175560 & 24,22 & 24175 & 26,28 & 1,13 & $1,12-1,15$ & $<0,0001$ \\
\hline \multirow[t]{2}{*}{ Diabetes } & No & 492302 & 67,92 & 60704 & 65,99 & 1 & & \\
\hline & Sí & 232496 & 32,08 & 31281 & 34,01 & 1,1 & $1,09-1,12$ & $<0,0001$ \\
\hline \multirow[t]{2}{*}{ TEP } & No & 723023 & 99,76 & 91571 & 99,55 & 1 & & \\
\hline & Sí & 1775 & 0,24 & 414 & 0,45 & 2,1 & $1,88-2,34$ & $<0,0001$ \\
\hline \multirow[t]{2}{*}{ Anemia } & No & 629545 & 86,86 & 75121 & 81,67 & 1 & & \\
\hline & Sí & 95253 & 13,14 & 16864 & 18,33 & 1,59 & $1,56-1,62$ & $<0,0001$ \\
\hline \multirow[t]{2}{*}{ Insuf. renal } & No & 630170 & 86,94 & 77351 & 84,09 & 1 & & \\
\hline & Sí & 94628 & 13,06 & 14634 & 15,91 & 1,31 & $1,28-1,33$ & $<0,0001$ \\
\hline \multirow[t]{2}{*}{ Arritmias } & No & 345673 & 47,69 & 43691 & 47,50 & 1 & & \\
\hline & Sí & 379125 & 52,31 & 48294 & 52,50 & 1,01 & 0,99-1,02 & 0,21 \\
\hline \multirow[t]{2}{*}{ Ictus } & No & 695289 & 95,93 & 87428 & 95,05 & 1 & & \\
\hline & Sí & 29509 & 4,07 & 4557 & 4,95 & 1,27 & $1,23-1,31$ & $<0,0001$ \\
\hline \multirow[t]{2}{*}{ Dislipemia } & No & 625278 & 86,27 & 81143 & 88,21 & 1 & & \\
\hline & Sí & 99520 & 13,73 & 10842 & 11,79 & 0,82 & $0,80-0,84$ & $<0,0001$ \\
\hline \multirow[t]{2}{*}{ C. Isquémica } & No & 531678 & 73,35 & 67850 & 73,76 & 1 & & \\
\hline & Sí & 193120 & 26,64 & 24135 & 26,24 & 0,98 & 0,96-0,99 & 0,003 \\
\hline
\end{tabular}

EP_vivos: estancia anormalmente prolongada y dado de alta vivo; TEP: tromboembolismo pulmonar; OR: odds ratio; IC 95\% OR: IC de confianza al $95 \%$ para la OR.

Los diagnósticos que con mayor fuerza se asociaron a EP fueron el TEP, anemia e IR; por el contrario presentaron menor probabilidad los que tenían codificaciones de HTA, dislipemia y CI.

\section{Principales comorbilidades según tres categorías al alta y totales. Análisis bivariado}

Para detallar algunos resultados aparentemente paradójicos del epígrafe pre- vio fueron estudiados los pacientes en tres grupos (EP_vivos, NoEP_vivos y exitus) de tal modo que pudiéramos aislar los exitus de los resultados. Se asociaron a ser EP_vivos los diagnósticos de EPOC, valvulopatías, miocardiopatías, anemia y DM. En el subgrupo de exitus fue más frecuente la asociación con ictus, IR y TEP. Los resultados se muestran con detalle en la tabla 3. 
Tabla 3. Presentación de las principales comorbilidades, reingresos y variables cuantitativas según tres categorías al alta y totales. Análisis bivariado

\begin{tabular}{|c|c|c|c|c|c|c|c|c|}
\hline & $\begin{array}{c}\text { NoEP } \\
\text { Vivos (\%) }\end{array}$ & IC95\% & $\underset{\text { Vivos(\%) }}{\text { PP }_{-}}$ & IC $95 \%$ & Exitus(\%) & IC95\% & Total & IC95\% \\
\hline EPOC & 18,9 & $18,8-18,9$ & 19,9 & $19,6-20,1$ & 18,1 & $17,9-18,4$ & 18,9 & $18,8-19,0$ \\
\hline C. Isquémica & 26,7 & $26,6-26,8$ & 26,2 & $26,0-26,5$ & 24,3 & $24,0-24,6$ & 26,4 & $26,3-26,0$ \\
\hline HTA & 49,7 & $49,5-49,8$ & 44,4 & $44,0-44,6$ & 36 & $35,7-36,4$ & 47,6 & $47,5-47,8$ \\
\hline Valvulopatías & 23,9 & $23,8-24,0$ & 26,3 & $26,0-26,6$ & 18,4 & $18,2-18,7$ & 23,6 & $23,5-23,7$ \\
\hline MCD & 10,9 & $10,8-10,9$ & 11,4 & $11,2-11,6$ & 8,6 & $8,4-8,8$ & 10,7 & $10,6-10,8$ \\
\hline Arritmias & 52,3 & $52,1-52,4$ & 52,5 & $52,2-52,8$ & 51,1 & $50,7-51,4$ & 52,2 & $52,1-52,3$ \\
\hline Ictus & 3,9 & $3,9-4,0$ & 5 & $4,8-5,1$ & 7,4 & $7,3-7,6$ & 4,4 & $4,3-4,5$ \\
\hline Insuf. Renal & 12,6 & $12,6-12,7$ & 15,9 & $15,7-16,1$ & 25,3 & $25,0-25,6$ & 14,3 & $14,2-14,4$ \\
\hline Anemia & 12,4 & $12,3-12,4$ & 18,3 & $18,1-18,6$ & 12,7 & $12,5-12,9$ & 13,1 & $13,0-13,2$ \\
\hline TEP & 0,2 & $0,20-0,23$ & 0,5 & $0,4-0,5$ & 1,5 & $1,4-1,6$ & 0,4 & $0,36-0,39$ \\
\hline $\mathrm{FA}$ & 44,8 & $44,6-44,9$ & 45,2 & $44,9-45,5$ & 37,8 & $37,5-38,2$ & 44,1 & $43,9-44,2$ \\
\hline Diabetes & 31,8 & $31,7-32,0$ & 34 & $33,7-34,3$ & 26,8 & $26,5-27,1$ & 31,5 & $31,4-31,6$ \\
\hline Dislipemia & 14 & $13,4-14,1$ & 11,8 & $11,6-12,0$ & 7,5 & $7,3-7,7$ & 13,1 & $13,0-13,2$ \\
\hline Reingresos & 10,2 & $10,1-10,3$ & 11,1 & $10,9-11,3$ & 14,3 & $14,1-14,6$ & 10,7 & $10,6-10,8$ \\
\hline Estancia en días & $6,92(3,34)$ & $6,93-9,91$ & $17,22(2,48)$ & $17,20-17,24$ & $6,52(5,65)$ & $6,48-6,56$ & $8,05(4,85)$ & $8,04-8,06$ \\
\hline № Diagnósticos & $6,61(2,85)$ & $6,60-6,62$ & $7,41(2,99)$ & $7,39-7,43$ & $6,74(3,04)$ & $6,72-6,77$ & $6,72(2,90)$ & $6,71-6,73$ \\
\hline № Procedimientos & $1,75(2,18)$ & $1,74-1,76$ & $2,59(2,63)$ & $2,57-2,61$ & $1,84(2,48)$ & $1,82-1,86$ & $1,85(2,28)$ & $1,84-1,86$ \\
\hline
\end{tabular}

NoEP_vivos: Estancia no prolongada dado de alta vivo; EP_vivos: estancias prolongadas dados de alta vivos; MCD: miocardiopatías; TEP: tromboembolismo pulmonar; FA: fibrilación auricular; O: los valores entre paréntesis corresponden a la desviación estándar respecto de la media.

En el modelo multivariante (Tabla 4) fueron detectados como factores asociados a una mayor probabilidad de ser EP en el subgrupo de pacientes dados de alta vivos el sexo masculino, haber ingresado de modo programado o reingresado y tener más de 6 diagnósticos o más de 2 procedimientos al alta. Las comorbilidades asociadas a mayor probabilidad de ser EP fueron el TEP, la anemia, IR y el ictus. 
FACTORES ASOCIADOS A LAS ESTANCIAS ANORMALMENTE PROLONGADAS EN LAS HOSPITALIZACIONES....

Tabla 4. Factores asociados a EP en pacientes dados de alta vivos. Regresión logística multivariante

\begin{tabular}{|c|c|c|c|c|}
\hline & & OR & IC 95\% OR & $\mathbf{p}$ \\
\hline \multirow{2}{*}{ Sexo } & Mujer & 1 & & \\
\hline & Hombre & 0,93 & $0,92-0,95$ & $<0,0001$ \\
\hline \multirow{2}{*}{ Tipo de ingreso } & Urgente & 1 & & \\
\hline & Programado & 1,19 & $1,16-1,22$ & $<0,0001$ \\
\hline \multirow{2}{*}{ Reingreso } & No & 1 & & \\
\hline & $\mathrm{Si}$ & 1,09 & $1,06-1,11$ & $<0,0001$ \\
\hline \multirow{4}{*}{ Edad, años } & $45-50$ & 1 & & \\
\hline & $86-90$ & 0,92 & $0,86-0,98$ & 0,01 \\
\hline & $91-95$ & 0,84 & $0,78-0,89$ & 0,00 \\
\hline & $>95$ & 0,80 & $0,72-0,89$ & 0,00 \\
\hline \multirow{4}{*}{ № Diagnósticos } & $0-1$ & 1,00 & & \\
\hline & $2-3$ & 0,81 & $0,75-0,88$ & $<0,0001$ \\
\hline & $4-6$ & 1,09 & $1,01-1,19$ & 0,02 \\
\hline & $>6$ & 1,26 & $1,16-1,36$ & $<0,0001$ \\
\hline \multirow{4}{*}{ № Procedimientos } & $0-1$ & 1,00 & & \\
\hline & $2-3$ & 1,55 & $1,52-1,58$ & $<0,0001$ \\
\hline & $4-5$ & 1,82 & $1,79-1,86$ & $<0,0001$ \\
\hline & $>5$ & 2,25 & $2,20-2,30$ & $<0,0001$ \\
\hline \multirow{2}{*}{ EPOC } & No & 1 & & \\
\hline & Sí & 1,04 & $1,02-1,06$ & $<0,0001$ \\
\hline \multirow{2}{*}{ Valvulopatías } & No & 1 & & \\
\hline & Sí & 1,02 & $1,00-1,04$ & 0,016 \\
\hline \multirow{2}{*}{ TEP } & No & 1 & & \\
\hline & Sí & 1,83 & $1,63-2,04$ & $<0,0001$ \\
\hline \multirow{2}{*}{ Anemia } & No & 1 & & \\
\hline & Sí & 1,36 & $1,34-1,39$ & $<0,0001$ \\
\hline \multirow{2}{*}{ Insuf. renal } & No & 1 & & \\
\hline & Sí & 1,21 & $1,18-1,23$ & $<0,0001$ \\
\hline \multirow{2}{*}{ Ictus } & No & 1 & & \\
\hline & Sí & 1,15 & $1,12-1,19$ & $<0,0001$ \\
\hline
\end{tabular}

$\mathrm{n}=724017$; C-statistics: 0,614; $\mathrm{p}$ (Hosmer-Lemeshow) $=0,001$.

Variables no significativas: No se encontraron

Variables no incluidas pese a significación: grupo hospitalario, año de ingreso.

OR: Odds Ratio. IC 95\% OR: intervalo de confianza al 95\% para la OR. 


\section{DISCUSIÓN}

El valor de las estancias hospitalarias de los pacientes ingresados por IC es innegable, tanto desde el punto de vista clínico como del empleo de recursos y el coste económico que ello representa ${ }^{5,9}$. Además, con la óptica de la gestión hospitalaria, la estancia es una variable proxy del coste directo $^{5}$. No obstante, la estancia, dentro de un mismo GRD, no siempre depende de las características del paciente, sino que puede estar relacionada con determinadas situaciones específicas de calidad y de gestión.

Detectar una subpoblación de pacientes que, a priori, pueda tener una mayor probabilidad de presentar estancias prolongadas permitiría optimizar determinados recursos e incluso habilitar otros no instaurados todavía en todos los centros -como la hospitalización a domicilio, las unidades de corta estancia dependientes de Urgencias, los programas de telemedici$\mathrm{na}^{10} \mathrm{o}$ soporte telefónico ${ }^{10,11}$, etc.- así como prevenir reingresos.

Trabajar con registros como el CMBD y agrupaciones de rúbricas bajo GRD presenta el conocido inconveniente de manejar datos estrictamente hospitalarios, y el deber de asumir las limitaciones en la calidad de las codificaciones -que se presuponen con una progresiva mejora de su calidad a lo largo del periodo estudiado- así como cierta tendencia a considerar la causa de la muerte como isquémica cuando el paciente con IC fallece como consecuencia de este evento final. Existen otras limitaciones importantes que dificultan seriamente el análisis de los casos cuando se manejan estas bases de datos, especialmente la existencia de un posible sesgo de información consistente en el infraregistro de las patologías crónicas de tal modo que los pacientes más graves y que fallecen en los primeros días tras el ingreso suelen tener un bajo registro de comorbilidades crónicas y un alto registro de aquellas patologías graves y agudas que ocasionan el ingreso y con frecuencia la muerte. Este fenómeno fue descrito en 1988 por Jencks y col ${ }^{12}$ y posteriormente se ha confirmado su exis- tencia en múltiples trabajos. Iezzoni y col $^{13}$ confirmaron su presencia aún ampliando el número de codificaciones diagnósticas (de 5 en el trabajo de Jencks a 25 en el de Iezzoni) y añadieron como otra posible explicación al fenómeno la incentivación por codificaciones (sistema de pago prospectivo según el cual se incentiva la inclusión de al menos un diagnóstico secundario), pero dicho sistema no mejora el pago por la adición de más diagnósticos.

En España hay varios trabajos de calidad que avalan la existencia en nuestro medio de dicho sesgo y que excluyen claramente como elemento causal (en el todo y en las partes) que el pago prospectivo tenga relación alguna con los resultados, dadas las características específicas de nuestro sistema sanitario. Los trabajos de Librero y col $^{14}$ y Peiró y col ${ }^{15}$ confirmaron en nuestro país que existen diagnósticos crónicos y graves que se comportan sistemáticamente como factores protectores frente a la mortalidad hospitalaria e insisten, creemos que acertadamente, en que la forma más eficiente de mejorar los registros consiste en fortalecer el número y calidad de las codificaciones de los médicos informantes, información de la que se nutre en definitiva la construcción del CMBD. En nuestro trabajo este efecto se ha intentado minimizar agrupando los pacientes en tres categorías (desagregando los exitus en un tercer grupo) lo que permite que diferencias estadísticamente significativas atribuibles al alto volumen muestral pero clínicamente muy poco relevantes como las mostradas al comparar los EP frente a los NoEP muestren, tras la estratificación, diferencias más consistentes y próximas a la realidad. Pese a ello hemos objetivado que la situación en cuanto a los sesgos comentados es superponible cuando se trata de analizar estancias prolongadas, habiendo obtenido prevalencias de HTA, CI y dislipemia sustancialmente superiores en los pacientes con menores estancias y que cursan alta vivos que en el resto de la población hospitalizada; también se ha podido confirmar que en la población de pacientes fallecidos las proporciones de dichas patologías crónicas son netamente inferiores 
al resto de la muestra (en sintonía con los trabajos previos). En contraposición a esto fue posible aislar tres comorbilidades asociadas a estancias prolongadas que presentan una mayor frecuencia de presentación a medida que los pacientes corresponden a grupos de mayor gravedad, esto es, son más frecuentes en lo exitus que en el resto, y también lo son en los que tienen estancias prolongadas frente a los que no las tienen (ictus, IR y TEP).

Otra importante limitación es la escasa capacidad discriminativa de los modelos de regresión, ya comprobada en trabajo previos sobre mortalidad ${ }^{15}$ y que puede estar en relación como bien se apunta en ellos con la falta de variables críticas, en el caso de la IC, la clase funcional y la fracción de eyección, entre otras. Finalmente en lo que respecta la calibración del modelo, la no existencia de significación tan sólo traduce un elevadísimo tamaño muestral pero las diferencias entre los valores observados y predichos en los deciles de riesgo del test de Hosmer-Lemeshow presentan diferencias que, a nuestro criterio, son aceptables.

Vistas las principales limitaciones, creemos que el trabajo con registros exhaustivos como el utilizado, permite enfrentarnos a la totalidad de los casos con la potencia muestral que ello confiere, evitando algunas limitaciones de otras publicaciones como la diferente proporción de inclusiones por sexo, ya que la mayor parte de los estudios presentan una escasa proporción de mujeres entre sus participantes ${ }^{16,17}$.

A lo largo de los 11 años estudiados se ha asistido a un crecimiento progresivo del GRD 544, traducido por casos con mayor coste-peso y, por ende, complejidad diagnóstico-terapéutica, posiblemente también relacionado con un mayor volumen de población anciana y con una mejor cumplimentación de datos y consiguiente codificación, aunque no se han hallado diferencias por sexos en cuanto a la distribución en cada GRD. También desde el punto de vista del género, la población femenina presenta con mayor frecuencia EP, y lo hace siguiendo una curva muy similar a la de la distribución según la edad en la preva- lencia de $\mathrm{IC}^{18-21}$ : en los grupos de edad más jóvenes predominan los varones y, por tanto, éstos aglutinan una mayor proporción de EP, pero este hecho se invierte a medida que las mujeres se hacen mayoritarias, merced a una mortalidad más tardía que la de los varones. Para ambos grupos de estancias, la edad media reportada en nuestro trabajo ha sido discretamente superior a otras publicaciones, como las del Registro RAIC $^{22}$, el BADAPIC ${ }^{23}$ o las del reciente estudio PRICE ${ }^{21}$, y similar a las citadas en el GALLICAP ${ }^{24}$.

Parece lógico que la mortalidad sea mayor durante los reingresos que en el resto de situaciones analizadas; no obstante, hay una diferencia clínicamente poco relevante en la proporción de reingresos que se da en los dados de alta vivos, independientemente de la estancia, lo cual nos hace plantearnos si el reingreso es realmente un mal indicador o por el contrario se tiende a valorar en exceso su trascendencia. La estancia media tuvo un comportamiento muy similar en el caso de los exitus y en aquellos dados de alta vivos; parece lógico que los NDA y NPA sean mayores a medida que aumenta la estancia, bajando de nuevo en el caso de los fallecidos por los motivos ya comentados.

En cuanto a las comorbilidades, tal como fue comentado la HTA y la CI disminuyen la probabilidad de pertenecer a los grupos EP, si bien su frecuencia de presentación por sexos es congruente con los hallazgos de la literatura ${ }^{16,17,20,21,23}$. Los pacientes con patologías que, a menudo, ocasionan descompensaciones de IC (anemia, insuficiencia renal, EPOC) y los reingresos previos sí se asocian con mayor frecuencia con EP. Además, HTA, EPOC, CI, valvulopatías, insuficiencia renal, anemia, diabetes, dislipemia y arritmias (fundamentalmente fibrilación auricular) aumentan porcentualmente durante los 11 años analizados, en tanto apenas lo hacen cardiopatías congénitas, ictus y miocardiopatías.

Si bien parece oportuno desarrollar trabajos especialmente diseñados para confirmar las hipótesis sustentadas y los resultados obtenidos, creemos que detec- 
tar al ingreso las variables asociadas a EP puede conducir a una práctica y un enfoque más eficientes de nuestros pacientes con IC. Alternativas estratégicas recientes -como la telemonitorización de patrones clínicos concretos (peso, tensión arterial, frecuencia cardíaca, diuresis), unidades específicas de IC y de corta estancia, dependientes de Urgencias, la coordinación sociosanitaria y la colaboración estrecha con la Atención Primaria o la ayuda telefónica, que reducen la admisión hospitalaria, la mortalidad y el coste sanitario- deberían ser introducidas ampliamente en el arsenal de cuidados actuales de salud ${ }^{10,11,25-27}$. Para finalizar, debemos insistir en que la mejora en el número y calidad de las codificaciones emitidas por los médicos informantes es, a nuestro criterio, la llave para poder explotar el gran potencial de este tipo de bases de datos.

\section{BIBLIOGRAFÍA}

1. Ho K, Pinsky J, Kannel W, Levy D. The epidemiology of heart failure: the Frammingham study. J Am Coll Cardiol 1993; 22: 4 Suppl A $6 \mathrm{~A}-13 \mathrm{~A}$.

2. Lloyd D, Larson M, Leip E, Beiser A, D’agostino $\mathrm{R}, \mathrm{KANNEL} \mathrm{W}$ et al. Lifetime risk for developing congestive heart failure: the Framingham Heart Study. Circulation 2002; 106: 29972998.

3. McMurray J, Stewart S. Epidemiology, aetiology and prognosis of heart failure. Heart 2000; 83: 596-602.

4. de la Fuente Cid R, Hermida A, Pazo M, Lado F. Epidemiología de la insuficiencia cardiaca. Porporciones de epidemia. An Med Interna (Madrid) 2007; 24: 500-504.

5. Banegas J, Rodríguez Artalejo J, Guallar P. Situación epidemiológica de la insuficiencia cardiaca en España. Rev Esp Cardiol 2006; 6: 4C-9C.

6. Cots F, Castells X, García A, Sanz M. Relación de los costes directos de la hospitalización con la duración de la estancia. Gac Sanit 1997; 11: 287-295.

7. CMBD años 1997-2007. Instituto de Información Sanitaria, Ministerio de Sanidad y Política Social. Madrid 2009.

8. Statistical Package for de Social Sciences (SPSS). En: 15 ed. Chicago: SPSS inc 2006.
9. Peiró S, Meneu de Guillerna R, Roselló ML, Martínez E, Portella E. ¿Qué mide la estancia media de los grupos relacionados de diagnóstico? Med Clin 1994; 103: 413-417.

10. Clark R, Inglis S, McAlister F, Cleland J, Stewart S. Telemonitoring or structured telephone support programmes for patients with chronic hear failure: systematic review and metaanalysis. Br Med J 2007; 334: 942-950.

11. Gamboa F, Gómez Camacho E, de Villar E, Vega J, Mayoral L, López R. Un nuevo modelo de cuidados médicos para los pacientes multiingresadores. Rev Clin Esp 2002; 202: 187-196.

12. Jencks S, Williams D, Kay T. Assesing hospital-associated deaths from discharge data. JAMA 1988; 260: 2240-2246.

13. IEzzonI Li. Risk adjustment for Medical Outcomes Studies. En: Grady ML, ed. Medical Effectiveness Research Data Methods. Rockville, MD: Agency for Health Care Policy and Research 1992: 83-97.

14. Librero J, Peiro S. ¿Previenen enfermedades crónicas la mortalidad de intrahospital? Paradojas y tendencias en información sobre morbosidad de hospital. Gac Sanit 1998; 12: 199-206.

15. Peiró S, Librero J, Ordiñana R. Perfiles de mortalidad hospitalaria: ¿una herramienta útil para la identificación de potenciales problemas de calidad? Rev Calidad Asistencial 1997; 12: 179-187.

16. Altimir S, Lupón J, González B, Prats M, Parajón T, URRUtia A et al. Sex and age differences in fragility in a heart failure population. Eur $\mathrm{J}$ Heart Fail 2005; 7: 798-802.

17. Mezu U, Bott Silverman C, Hisch E. Heart failure in women is different than in men: should treatment be different? Clevel Clin J Med 2007; 74: 423-435.

18. CowIE MR. Annotated references in epidemiology. Eur J Heart Fail 1999; 1: 101-107.

19. Rodríguez J, Banegas J, Guallar P. Epidemiología de la insuficiencia cardiaca. Rev Esp Cardiol 2004; 57:163-170.

20. Cortina A, Reguero J, Segovia E, Rodríguez Lambert J, Cortina R, ARias JC et al. Prevalence of heart failure in Asturias (A Region in the North of Spain). Am J Cardiol 2001; 87: 14171419.

21. Anguita M, Crespo MG, de Teresa Galván E, JiméNEZ M, ALonso L, MuÑIZ J. Prevalencia de la insuficiencia cardiaca en la poblacion general española mayor de 45 años. Estudio PRICE. Rev Esp Cardiol 2008; 61: 1041-1049. 
22. Jiménez MF, Garcia J, Anguita M, Martínez A, Torres F. Investigadores del registro RAIC. Características demográficas de los pacientes ingresados por insuficiencia cardiaca en Andalucía: resultados del estudio RAIC. Rev And Cardiol 2006; 41:107-113.

23. Anguita M. Características clínicas, tratamiento y morbimortalidad a corto plazo de pacientes con insuficiencia cardiaca controlados en consultas específicas de insuficiencia cardiaca. Resultados del registro BADAPIC. Rev Esp Cardiol 2004; 57: 1159-1169.

24. Otero F, Grigorian L, Fransi L, Názara C, FernánDEZ JM, del Alamo A et al. Morbidity and mortality among heart failure patients in Galicia, N.W. Spain: the GALICAP study. Int J Cardiol 2009; 136: 56-63.
25. Seto E. Cost comparison between telemonitoring and usual care of heart failure: a systemactic review. Telemed JE Health 2008; 14: 679-686.

26. Chaudhry S, Wang Y, Concato J, Gill T, Krumholz H. Patterns of weight change preceding hospitalization for heart failure. Circulation 2007; 116: 1549-1554.

27. Weintraub NL, Collins SP, Pang PS, Levy PD, Anderson AS, Arslanian-Engoren A et al. Acute heart failure syndromes: Emergency Department presentation, treatment and disposition: current approaches and future aim. A scientific statement from the American Heart Association. Circulation 2010; doi: 10.1161/CIR.0b013e3181f9a223. 